\title{
Flat (001) surfaces of II-VI semiconductors: A lattice gas model
}

\author{
Martin Ahr*, Michael Biehl \\ Institut für Theoretische Physik und Astrophysik \\ Julius-Maximilians-Universität Würzburg \\ Am Hubland, D-97074 Würzburg, Germany
}

October 29,2018

\begin{abstract}
We present a two-dimensional lattice gas with anisotropic interactions which model the known properties of the surface reconstructions of CdTe and ZnSe. In contrast to an earlier publication [12] the formation of anion dimers is considered. This alters the behaviour of the model considerably. We determine the phase diagram of this model by means of transfer matrix calculations and Monte Carlo simulations. We find qualitative agreement with the results of various experimental investigations.
\end{abstract}

Keywords: Equilibrium thermodynamics and statistical mechanics, Monte Carlo simulations, Surface relaxation and reconstruction, Surface thermodynamics (including phase transitions), Cadmium telluride, Zinc selenide, Low index single crystal surfaces.

\section{Introduction}

Within the last years, potential technological applications of electronic devices based on IIVI semiconductors [1] have inspired basic research concerning surfaces of these materials. In this context, various studies have addressed the properties of surface reconstructions. Experimental studies have investigated which reconstructions are present [2, 3] and how the reconstruction of the surface is influenced by parameters like temperature and particle flux in an MBE environment [4, 5, 6]. The majority of this work has been devoted to CdTe and $\mathrm{ZnSe}$, where a fairly complete qualitative overview over the phase diagram has been gained. An overview over the properties of CdTe can be found in [7]. On the other hand, there have been theoretical investigations of the reconstructions of $\mathrm{CdTe}[8]$ and $\mathrm{ZnSe}$ [9, 10] using density functional theory. In these studies, knowledge about the chemical bonding of surface atoms and ground state energies of various reconstructions has been gained.

Being based on quantum mechanics, density functional theory is believed to be exact apart from approximations made in the calculation. The computational burden of this method is comparatively high, which restricts its practical applicability to systems consisting of only a few atoms. Due to the periodicity of crystal surfaces this is not a severe

${ }^{*}$ Corresponding author. Phone: +49 (0)931 8884908 Fax: +49 (0)931 8885141 E-mail ahr@physik.uniwuerzburg.de 


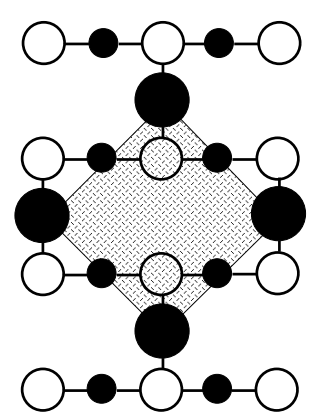

(a)

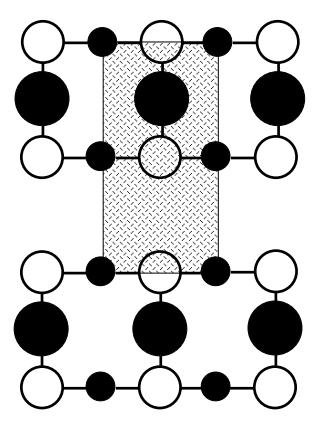

(b)

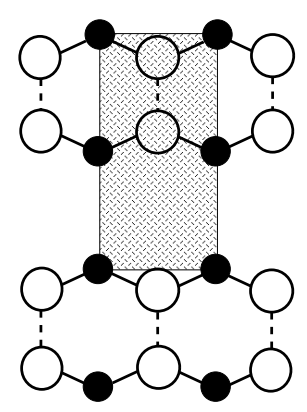

(c)

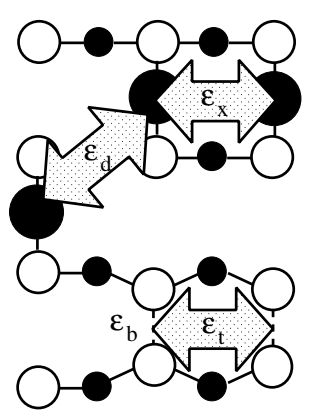

(d)

Figure 1: Panels (a),(b),(c): Sketches of the reconstructions of CdTe which are discussed in this work. The grey rectangles show the surface unit cells. The [110] axis is aligned horizontally. (a), (b): Cd-terminated reconstructions. Panel (a) shows the $c(2 \times 2)_{\mathrm{Cd}}$ reconstruction, panel $(\mathrm{b})$ the $(2 \times 1)_{\mathrm{Cd}}$ reconstruction. Panel $(\mathrm{c})$ shows the $(2 \times 1)_{\mathrm{Te}}$ reconstrution of surfaces terminated with a complete monolayer of Te. Panel (d) shows the attractive couplings in our model.

restriction if one is interested in ground state properties of the system which however, are strictly relevant only at zero temperature. At higher temperature, the properties of the surface will be influenced by thermodynamic effects. These are particularly important if phase transitions between different reconstructions occur. Their theoretical investigation requires the study of systems with a large number of atoms which is beyond the scope of first principles methods or molecular dynamics simulations using realistic empirical potentials.

Consequently, simplifying models are needed which preserve essential features of atomic interactions and can be investigated with moderate numerical effort. In many cases, two-dimensional lattice gases have been used successfully to model atoms adsorbed on a singular crystal surface or the terminating layer of such a crystal [11, 12, 13, 14]. In spite of the conceptual simplicity of such models the interplay of attractive and repulsive short range interactions can result in highly nontrivial critical behaviour and complex phase diagrams. In this paper, we will follow this approach to model the reconstructions of (001) surfaces of CdTe and ZnSe, our main focus being on CdTe.

The outline of this paper is as follows: In section 2, we will give a short review of the known facts about the reconstructions of the (001) surfaces of CdTe and ZnSe. In section 3, we introduce a lattice gas model which considers the occupation of $\mathrm{Cd}$ sites and the dimerization of Te atoms and discuss its phase diagram. We conclude with a comparison of the features of our model with experimental results in section 4 .

\section{Surface reconstructions of CdTe and $\mathrm{ZnSe}$}

Both CdTe and ZnSe crystallize in the zinc-blende lattice. This lattice structure is composed of alternating layers of cations and anions which are parallel to the (001) surface, such that an ideal (001) surface would be terminated by a complete layer of one particle species. The positions of the atoms in one layer lie on a regular square lattice with its axes oriented in the $[110]$ direction and the $[1 \overline{1} 0]$ direction.

Under vacuum, the (001) surface of CdTe is Cd terminated. The surface is characterized by vacancy structures where less than one half of the potential $\mathrm{Cd}$ sites in the top 
layer are occupied [5, 6]. This can be understood from simple quantum-mechanical considerations like the electron counting rule [15, 16], which states that a surface terminated by a complete layer of $\mathrm{Cd}$ is energetically unfavourable. At low temperature, one finds a $c(2 \times 2)_{\mathrm{Cd}}$ reconstruction [7, 2], where $\mathrm{Cd}$ atoms and vacancies arrange in a checkerboard pattern (figure 1 1a). Frequently, a contribution of a $(2 \times 1)_{\mathrm{Cd}}$ arrangement can be found. In the $(2 \times 1)_{\mathrm{Cd}}$ structure, the Cd atoms arrange in rows along the [110] direction which alternate with rows of vacancies (figure 1 $1 \mathrm{~b}$ ). Density functional calculations [8] have shown that the surface energies of the two competing reconstructions are nearly degenerate and differ only by a small amount $\Delta E \approx 0.016 \mathrm{eV}$ per $(1 \times 1)$ surface unit cell. At a temperature $T \approx 570 K$, there is a phase transition [2] above which the $(2 \times 1)_{\mathrm{Cd}}$ arrangement of the $\mathrm{Cd}$ atoms dominates the surface. An analysis of high resolution low energy electron diffraction (HRLEED) peaks [3] has shown, that there is a high degree of disorder in this high temperature phase. One finds elongated domains with a large correlation length of $\approx 375 \AA$ in the $[1 \overline{1} 0]$ direction and a domain width as small as $22 \AA$ in the [110] direction.

If CdTe is exposed to an external Cd flux in an MBE chamber, the $c(2 \times 2)_{\mathrm{Cd}}$ reconstruction is stabilized at temperatures above the transition in vacuum. Under a Te flux, the surface is Te terminated with a $(2 \times 1)_{\mathrm{Te}}$ reconstruction. At small Te fluxes, the surface is terminated by a complete monolayer of Te. The Te atoms on the surface form dimers which arrange in rows (figure 1 $\mathrm{c}$ ). At high Te flux and low temperature, one additional Te atom is incorporated into each dimer, such that the Te coverage of the surface is 1.5. The symmetry of this reconstruction is still $(2 \times 1)$, since the Te trimers tend to arrange in rows. A schematical phase diagram of the surface can be found in []].

Qualitatively, the properties of the reconstructions of ZnSe [17, [1] are quite similar to those of $\mathrm{CdTe}$, where the $\mathrm{Zn}$ atoms are the counterparts of the $\mathrm{Cd}$ atoms and the Se atoms those of Te, respectively. There is one important exception: to date, no $(2 \times 1)_{\mathrm{Zn}}$ reconstructed surface has been found at high temperature. Density functional calculations [9, 10] yield a higher energy difference $\Delta E \approx 0.03 \mathrm{eV}$ per $(1 \times 1)$ surface unit cell between ideal $c(2 \times 2)$ and $(2 \times 1)$ reconstructed surfaces, which is approximately twice the value calculated for CdTe. As we will show, this greater energy difference might explain the different behaviour of CdTe and ZnSe.

\section{The lattice gas model}

The basic structure of our model of (001) surfaces is the same for different II-VI semiconductors. The differences between the materials are represented by the numerical values of parameters. In the following, for simplicity we will loosely speak of $\mathrm{Cd}$ and $\mathrm{Te}$ atoms instead of "cations" and "anions" without restricting ourselves to a modelling of CdTe only.

In the lattice gas picture, a crystal lattice which is fixed in space is considered. Each lattice site is either occupied by an atom or empty. There are effective interactions between atoms which include effects of surface strain and, at elevated temperature, lattice vibrations. Therefore, there is no simple mapping between ground state energies of the lattice gas model and surface energies determined from density functional theory.

We model a flat (001) surface of CdTe i.e. we neglect the influence of steps on the reconstruction. This is a reasonable approximation if the typical distance between steps is much greater than the size of the unit cell of the reconstruction. In thermal equilibrium, this is fulfilled at temperatures well below the roughening transition. In this case, in the absence of bulk vacancies or other defects the crystal is uniquely described by the state 
of the topmost monolayer which consists of one Cd half-layer and a Te half-layer. In our model, we consider such a monolayer with the Cd atoms on top. For simplicity, we assume the Te layer to be fully occupied. This is not a severe restriction, since the removal of Te atoms will uncover $\mathrm{Cd}$ atoms in the layer below. Within the limit of a model of a flat surface, we simply do not distinguish whether a $\mathrm{Cd}$ terminated surface has been created by removing a Te layer or by depositing additional $\mathrm{Cd}$ atoms onto an intact Te layer.

We use cartesian coordinates where the $x$-axis points in the [110] direction, the $y$-axis points in the [1피 direction and the $z$-axis points in the [001] direction. The origin of the coordinate system is at the center of a $\mathrm{Cd}$ atom. Since we consider a flat surface, the $z$-coordinate of all atoms of one species is identical and will be omitted in the following. Measuring the lattice constant in appropriate units, the $\mathrm{Cd}$ atoms are at positions $(x, y)$ with integer $x, y$. The Te atoms are at positions $(x, y+1 / 2)$, where $x, y \in \mathbb{Z}$. Tellurium dimers are created by the formation of a chemical bond between neighbouring Te atoms in $y$-direction $([1 \overline{1} 0])$. Since this is also the direction of the bonds of a surface Cd atom, a Te dimer is formed by a pair of atoms, which might also be the binding partners of a $\mathrm{Cd}$ atom above them. This suggests a simple lattice gas representation both of the occupation of $\mathrm{Cd}$ sites and Te dimerization: We consider a rectangular array $x$ of integers $\left\{x_{i, j}\right\}_{i, j=1}^{L, N} . x_{i, j}=1$ represents a Cd atom at site $(i, j)$, which is bound to the Te atoms at sites $(i, j-1 / 2)$ and $(i, j+1 / 2) . \quad x_{i, j}=2$ corresponds to a dimerization of Te atoms $(i, j-1 / 2)$ and $(i, j+1 / 2)$. Otherwise, $x_{i, j}=0$.

In principle, one might also consider the formation of Te trimers by introducing a fourth state $x_{i, j}=3$. This corresponds to a trimer which consists of the Te atoms at $(i, j-1 / 2)$ and $(i, j+1 / 2)$ and an additional Te atom at $(i, j)$. However, the formation of Te trimers plays a role only if the surface is exposed to a strong flux of pure Te at comparatively low temperature. Therefore, we have neglected this effect to reduce the number of parameters of our model and the numerical effort of its investigation.

\subsection{Interactions of atoms and dimers}

The detailed representation of the surface energies of a II-VI compound certainly would require long-range interactions and terms which depend on the simultaneous occupation of three or more sites. However, it is plausible to assume that the dominant contribution to the surface energy stems from pairwise interactions of atoms at short distances. In this section, we introduce a Hamiltonian which considers pairwise interactions between $x_{i, j}$ on nearest neighbour sites and diagonal neighbour sites. These reflect the known properties of the reconstructions of CdTe and ZnSe.

Due to the electron counting rule, surfaces with Cd coverages greater than $1 / 2$ are unstable while both a $c(2 \times 2)_{\mathrm{Cd}}$ reconstruction and a $(2 \times 1)_{\mathrm{Cd}}$ reconstruction are permitted. This feature can be captured by introducing a hardcore repulsion between $\mathrm{Cd}$ atoms on neighbouring sites in the $y$-direction. In the $x$-direction, an attractive interaction favours the occupation of nearest neighbour pairs the strength of which is denoted by $\epsilon_{x}<0$. An attractive interaction $\epsilon_{d}<0$ between diagonal neighbours stabilizes the $c(2 \times 2)_{\mathrm{Cd}}$ reconstruction. These parameters are chosen such that the energy difference $\Delta E=\left|2 \epsilon_{d}-\epsilon_{x}\right| / 2$ between these two reconstructions is small compared to the total surface energy per site.

The electron counting rule favours Te dimerization, but forbids the formation of additional bonds of dimerized Te atoms. Therefore, we forbid the simultaneous occupation of neighbour sites in the y-direction with dimers (formation of chains of Te-Te-bonds) and $\mathrm{Cd}$ atoms next to a dimer. These rules permit both a $(2 \times 1)_{\mathrm{Te}}$ reconstruction, where the dimers arrange in rows and a $c(2 \times 2)_{\mathrm{Te}}$ reconstruction, where they arrange in a checker- 
board pattern. Density functional calculations [8] have shown that the surface energy of $(2 \times 1)_{\mathrm{Te}}$ is significantly lower than that of a $c(2 \times 2)_{\mathrm{Te}}$ reconstruction. This may be understood from the more efficient relaxation of surface strain in $(2 \times 1)_{\mathrm{Te}}$ : Since the lattice is deformed in the same direction by dimers on neighbouring sites in x-direction, such an arrangement will be energetically favourable. We consider this fact by an attractive nearest neigbour interaction $\epsilon_{t}$ between dimers in x-direction. Additionally, a dimer contributes a binding energy $\epsilon_{b}$ to the surface energy. Apart from the hardcore repulsion, we consider no interaction between $\mathrm{Cd}$ atoms and dimers. An overview over the couplings in our model can be found in figure $1 \mathrm{~d}$.

This yields the Hamiltonian

$$
H=\sum_{i, j=1}^{L, N} \epsilon_{x} c_{i, j} c_{i+1, j}+\epsilon_{d} c_{i, j}\left(c_{i+1, j-1}+c_{i+1, j+1}\right)+\epsilon_{t} d_{i, j} d_{i+1, j}+\epsilon_{b} d_{i, j}-\mu c_{i, j},
$$

where we have introduced $c_{i, j}=\delta_{x_{i, j}, 1}$ and $d_{i, j}=\delta_{x_{i, j}, 2}$. The boundary conditions are assumed to be periodic. $c_{i, j}$ represents the occupation of lattice sites with $\mathrm{Cd}$ atoms: $c_{i, j}=1$ at the positions of $\mathrm{Cd}$ atoms and zero otherwise. Similarely, the positions of dimers are given by $d_{i, j}=1 . \quad \mu$ is the effective Cadmium chemical potential, which includes the binding energy of surface $\mathrm{Cd}$ atoms to the substrate. The groundstate of the system at $T=0$ is determined by the chemical potential $\mu$. For $\mu>\mu_{0}=2 \epsilon_{d}-\epsilon_{t}-\epsilon_{b}$, the surface configuration with minimal energy is $c(2 \times 2)_{\mathrm{Cd}}$. For $\mu<\mu_{0}$ the groundstate is $(2 \times 1)_{\mathrm{Te}}$.

In the following, we measure energy in dimensionless units which have been adjusted such that $\epsilon_{d}=-1$. Additionally, we set $k_{B}=1$ such that temperature is measured in units of $\left|\epsilon_{d}\right|$.

\subsection{Characterization of the surface configuration}

To characterize the surface reconstruction quantitatively, we evaluate the mean Cd coverage $\rho_{C d}=\left\langle c_{i, j}\right\rangle_{i, j}$ and the correlations

$$
\begin{aligned}
C_{C d}^{d} & =\frac{1}{2}\left\langle c_{i, j}\left(c_{i+1, j+1}+c_{i+1, j-1}\right)\right\rangle_{i, j} \\
C_{C d}^{x} & =\left\langle c_{i, j} c_{i+1, j}\right\rangle_{i, j} .
\end{aligned}
$$

$C_{C d}^{d}$ measures the probability to find two diagonal neighbour sites which are simultaneously occupied by $\mathrm{Cd}$ atoms. This is a measure for the fraction of the surface which is covered by regions with a local $c(2 \times 2)_{\mathrm{Cd}}$ order. Similarely, $C_{C d}^{x}$ measures the contribution of locally $(2 \times 1)_{\mathrm{Cd}}$ reconstructed regions in the system.

The long range order of the Cd atoms is measured by the order parameters

$$
\begin{aligned}
M_{C d}^{(2 \times 2)} & =\frac{1}{L N} \sum_{i, j=1}^{L, N} c_{i, j} \cos (\pi(i+j)) \\
M_{C d}^{(2 \times 1)} & =\frac{1}{L N} \sum_{i, j=1}^{L, N} c_{i, j} \cos (\pi j) .
\end{aligned}
$$

$M_{C d}^{(2 \times 2)}$ is the staggered magnetization of a system of Ising variables $\left\{s_{i, j}\right\}_{i, j=1}^{L, N}$, where $s_{i, j}=1$ if $c_{i, j}=1$ and -1 otherwise. Large absolute values indicate a long range order 
of the $c(2 \times 2)_{\mathrm{Cd}}$ reconstruction. Its counterpart $M_{C d}^{(2 \times 1)}$ measures the long range order of $(2 \times 1)_{\mathrm{Cd}}$.

Further, we introduce the mean Tellurium dimerization $\rho_{D}:=\left\langle d_{i, j}\right\rangle_{i, j}$ and the dimer correlation $C_{D}^{x}:=\left\langle d_{i, j} d_{i+1, j}\right\rangle_{i, j}$, which characterizes the number of dimers which are incorporated in locally $(2 \times 1)$ ordered areas. Their long range order is characterized by

$$
M_{D}^{(2 \times 1)}=\frac{1}{L N} \sum_{i, j=1}^{L, N} d_{i, j} \cos (\pi j) .
$$

\subsection{Methods of investigation}

We have investigated this model by means of the transfer matrix method and Monte Carlo simulations. An introduction to the transfer matrix technique can be found e.g. in [18, 21]. This method allows for a numerical calculation of the free energy of lattice systems with short-range interactions. In general, the system size must be finite in all directions but one. In our investigations, we choose the $y$-direction as the infinite direction. Since the computational effort increases exponentially fast with the system size $L$ in the $x$-direction, only comparatively small $L \leq 10$ are feasible. However, this is sufficient to investigate the phase transitions of the system in the limit $L \rightarrow \infty$ via finite size scaling techniques. To this end, we have applied the method suggested in [20] to determine the loci of first order phase transitions and to compute coverage discontinuities. Continuous transitions have been investigated by means of phenomenological renormalization group theory [19, 21].

Additionally, we have performed Monte Carlo simulations of our model. To obtain a reasonably fast equilibration, we have applied continuous time algorithms [22]. A more detailled description of these methods is presented in the appendix. We have simulated both the grand-canonical ensemble where $\rho_{C d}$ is controlled by $\mu$ and the canonical ensemble where the number of $\mathrm{Cd}$ atoms in the system is fixed. In general, the results of the transfer matrix calculations and the Monte Carlo simulations are in good agreement (figure 2).

\subsection{Results}

To get insight into the typical behaviour of the model, we will first present a detailed investigation using the parameter set $\epsilon_{d}=\epsilon_{b}=-1, \epsilon_{x}=\epsilon_{t}=-1.9$. The choice $\epsilon_{x}=-1.9$ reflects the fact that the energy difference between $c(2 \times 2)_{\mathrm{Cd}}$ and $(2 \times 1)_{\mathrm{Cd}}$ is small. The choice $\epsilon_{b}=-1$ yields low concentrations of Te atoms which are neither dimerized nor bound to $\mathrm{Cd}$ atoms. This is consistent with experimental results. For simplicity, we have chosen $\epsilon_{t}=\epsilon_{x}$ as a typical example of the case where both couplings are of the same order of magnitude. However, since there is no next-nearest neighbour interaction between dimers, there is a significant difference in the surface energies of $(2 \times 1)_{\mathrm{Te}}$ and $c(2 \times 2)_{\mathrm{Te}}$, which is consistent with the results of [8].

In section 3.4.4 we discuss the influence of the numerical values of the parameters on the phase diagram. We will show, that a smaller value of $\epsilon_{t}$ affects the phase diagram qualitatively. In particular, this concerns the high temperature phases.

\subsubsection{Grand-canonical ensemble}

With the above parameter set, the $\mathrm{Cd}$ and the Te terminated groundstates are separated by $\mu_{0}=0.9$. In figure 2 the temperature dependent behaviour of the system at constant chemical potential is shown for $\mu=1$ (figure 2a a,b) and $\mu=0.8$ (figure 2 ac,d), which are 

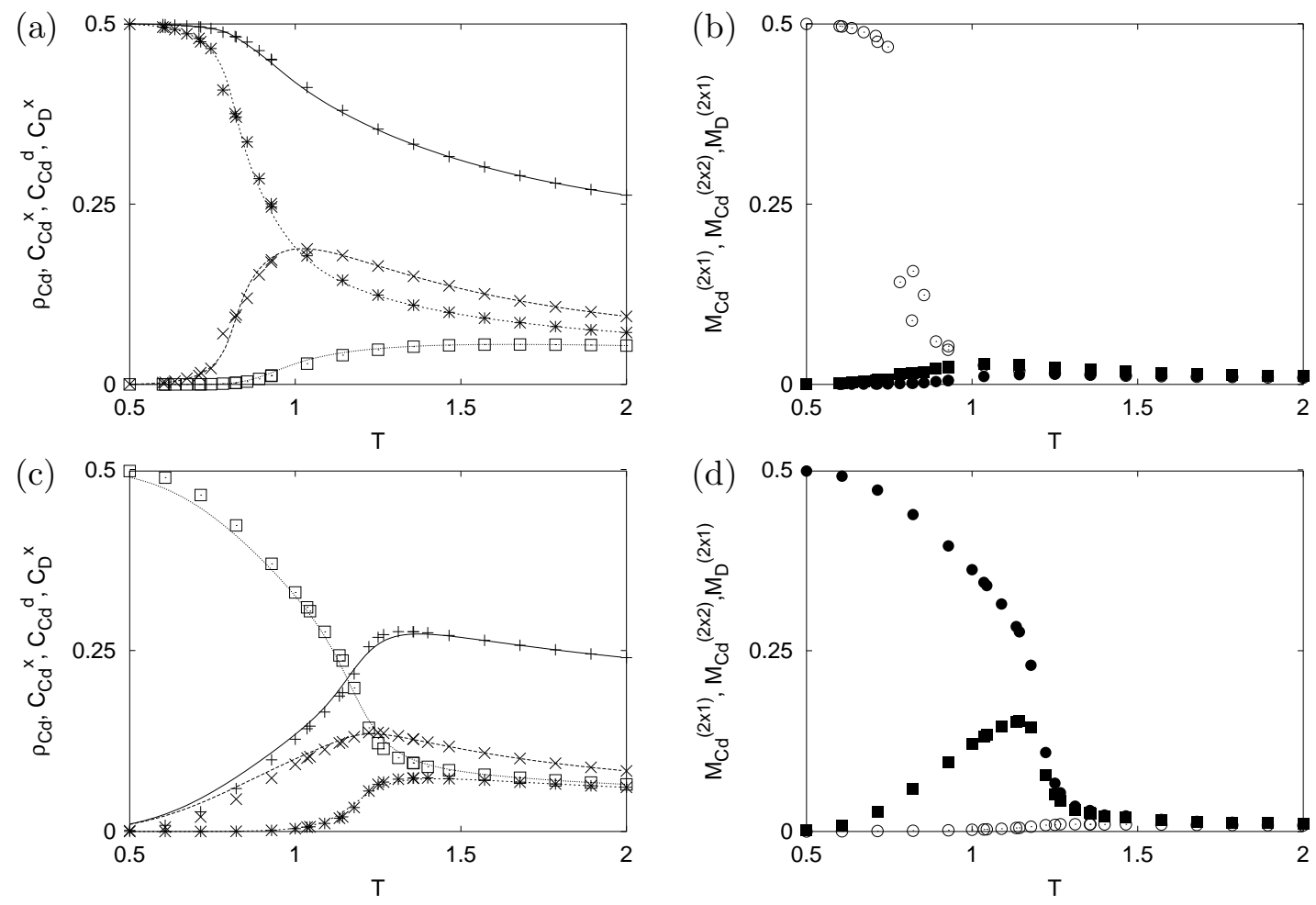

Figure 2: Simulations at constant chemical potential. Panels (a), (b) show the behaviour of the system with the couplings $\epsilon_{d}=\epsilon_{b}=-1, \epsilon_{x}=\epsilon_{t}=-1.9$ and a cadmium chemical potential $\mu=1$. With these parameters, the ground state of the system is $c(2 \times 2)_{\mathrm{Cd}}$. Panel (a) shows the Cd coverage $\rho_{C d}(+)$ and the correlations $C_{C d}^{x}(\times), C_{C d}^{d}(*)$ and $C_{d}^{x}(\square)$. Panel (b) shows the mean absolute of the order parameters $M_{C d}^{(2 \times 2)}(\odot), M_{C d}^{(2 \times 1)}(\mathbf{\square})$ and $M_{D}^{(2 \times 1)}(\bullet)$. The symbols show data from a simulation run at a system size $L=N=64$. $4 \cdot 10^{4} \cdot L N$ events have been performed both for equilibration and for measurement. The lines have been obtained by means of a transfer matrix calculation with a strip width $L=10$. The data shown in panels (c), (d) have been obtained at $\mu=0.8$, where the ground state is $(2 \times 1)_{\mathrm{Te}}$. All other parameters are identical to those used in (a), (b). 
examples for both cases. At $\mu=1$, the ordered $c(2 \times 2)_{\mathrm{Cd}}$ phase at low $T$ manifests itself in a high Cd coverage $\rho_{C d}$ and values of the correlation $C_{C d}^{d}$ and the order parameter $M_{C d}^{(2 \times 2)}$ close to 0.5 . In figure $3 \mathrm{~d}$ a surface snapshot in this phase at $T=0.71$ is shown. As expected, the $\mathrm{Cd}$ atoms arrange preferentially in a checkerboard configuration. At a temperature $T=0.83$, there is a first order phase transition to a disordered phase. This is indicated by a decrease of the order parameter $M_{C d}^{(2 \times 2)}$ and the correlation $C_{C d}^{d}$. Simultaneously, $C_{C d}^{x}$ starts to increase. At $T=1$, both lines cross such that the high temperature behaviour of the system is dominated by a local $(2 \times 1)$ ordering of the $\mathrm{Cd}$ atoms. The simulation data shown in figure $2 a, b$ have been taken from two independent simulation runs. The system was initialized with a perfect $c(2 \times 2)_{\mathrm{Cd}}$ configuration at the lowest temperature investigated. Then, as successively higher temperatures were imposed, the surface configuration was kept as initial state of the next simulation. This reflects in a small hysteresis effect in $M_{C d}^{(2 \times 2)}$ due to the first order nature of the phase transition.

At $\mu=0.8$, we obtain a completely different behaviour. Since the groundstate is a $(2 \times 1)_{\mathrm{Te}}$ reconstruction, at small $T$ we measure low $C d$ coverages and values of $C_{D}^{x}$ and $M_{D}^{(2 \times 1)}$ close to 0.5 . The most frequent thermal excitations are $\mathrm{Cd}$ adatoms, the density $\rho_{C d}$ of which increases with $T$. Figure 3c shows a surface snapshot at $T=0.93$. The $\mathrm{Cd}$ atoms preferentially arrange in rows such that $C_{C d}^{x} \gg C_{C d}^{d}$. These rows adapt to the structure of the $(2 \times 1)_{\mathrm{Te}}$ reconstruction. This yields nonzero values of the order parameter $M_{C d}^{(2 \times 1)}$, which indicate a global ordering of Cd atoms in a $(2 \times 1)$ arrangement. However, the interactions between the $\mathrm{Cd}$ atoms themselves are insufficient to stabilize this global order. Instead, it is purely induced by the environment of the Te dimers.

At $T=1.67$, there is a first order phase transition above which the system is in a disordered phase similar to that found at $\mu=1$. Remarkably, we observe high $\rho_{C d} \approx 0.2$ at temperatures slightly below the phase transition. At the phase transition, $\rho_{C d}$ jumps to an even higher value. Above the transition it decreases slightly with $T$.

\subsubsection{Phase diagram}

Figure 3a,b shows the phase diagram of the model, which has been extrapolated from transfer matrix calculations with strip widths $L$ of 6,8 and 10 lattice constants. In figure 3 $\mathrm{b}$, the lines of phase transitions in the $\mu-T$ plane have been plotted. At low temperature, the system is either in an ordered $(2 \times 1)_{\mathrm{Te}}(1)$ or an ordered $c(2 \times 2)_{\mathrm{Cd}}$ phase $(2)$. The line of phase transition between these phases starts at at zero temperature and $\mu=\mu_{0}$, where the energies of both reconstructions are degenerate. For $0<T<T_{t}=0.84$, it remains at the same chemical potential $\mu_{0}$, apart from small numerical uncertainties of extrapolation. In consequence, a phase transition between a $c(2 \times 2)_{\mathrm{Cd}}$ and a $(2 \times 1)_{\mathrm{Te}}$ reconstruction cannot be observed at constant chemical potential. The disordered phase (3) exists for $T \geq T_{t}$. At the point $\left(T=T_{t}, \mu=\mu_{0}\right)$ five phases coexist: the disordered phase, and $c(2 \times 2)_{\mathrm{Cd}}$ and $(2 \times 1)_{\mathrm{Te}}$ reconstructed phases in two sublattices, corresponding to positive and negative values of the order parameters $M_{C d}^{(2 \times 2)}$ and $M_{D}^{(2 \times 1)}$, respectively. The $(2 \times 1)_{\mathrm{Te}}$ reconstructed phase (1) exists only at temperatures below a critical temperature $T_{c}^{1}=\epsilon_{t}$. At $T_{c}^{1}$, the line of the phase transition to the disordered phase (3) diverges to $\mu=-\infty$. On the contrary, the $c(2 \times 2)_{\mathrm{Cd}}$ reconstructed phase (2) may exist at arbitrary temperature, if the $\mathrm{Cd}$ chemical potential is large enough. The dashed line in figure $3 \mathrm{~b}$ shows the chemical potential, at which the correlations $C_{C d}^{d}$ and $C_{C d}^{x}$ in the disordered phase are equal. For smaller $\mu$, the local ordering of $\mathrm{Cd}$ atoms is dominated by a $(2 \times 1)$ arrangement, while for larger $\mu$ they prefer a local $c(2 \times 2)$ ordering. 

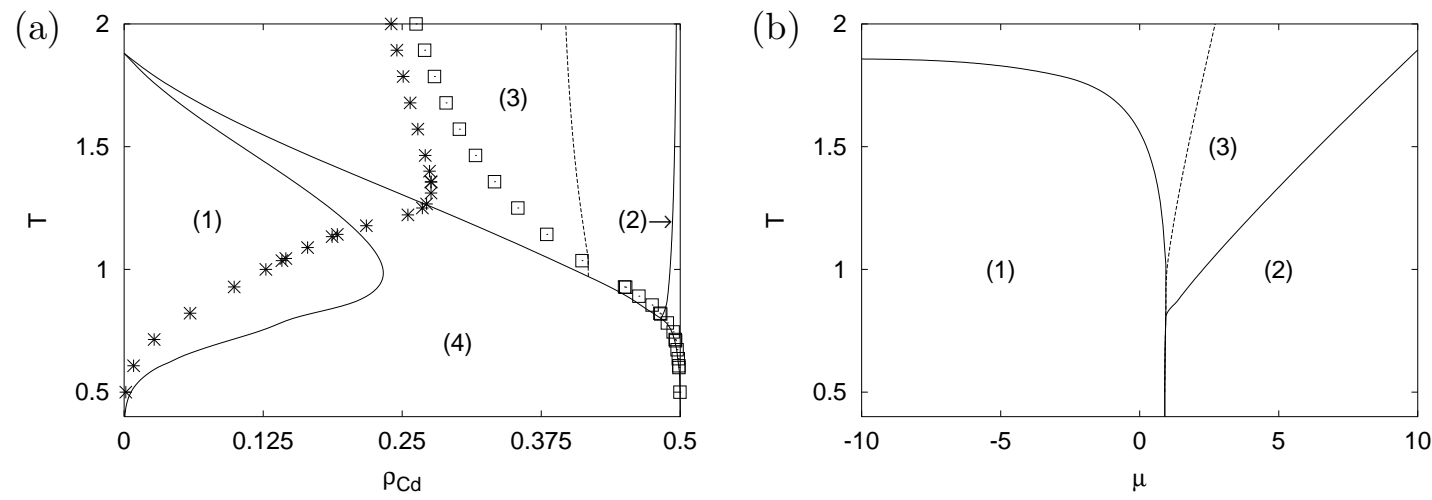

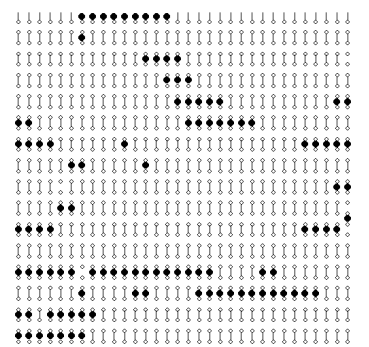

(c)

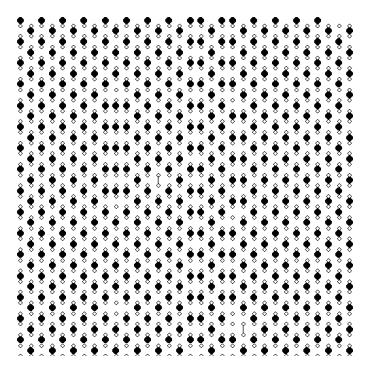

(d)

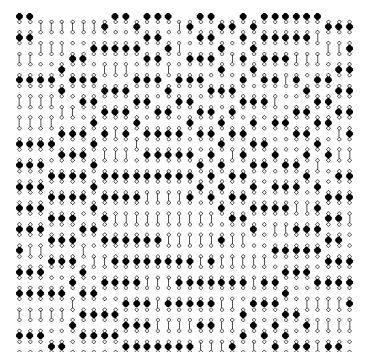

(e)

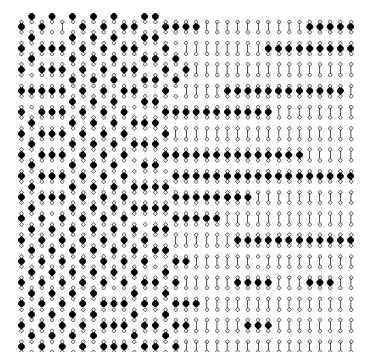

(f)

Figure 3: Phase diagram with parameters $\epsilon_{d}=\epsilon_{b}=-1, \epsilon_{x}=\epsilon_{t}=-1.9$. The solid lines in panel (a) show the lines of phase transitions as functions of $\rho_{C d}$ and $T$, in panel (b) they are shown in the $\mu-T$ plane. Note the offset in the temperature axes. In region (1), the system is in a homogeneously ordered phase with a $(2 \times 1)_{\mathrm{Te}}$ reconstruction, in region (2) it is homogeneously ordered and $c(2 \times 2)_{\mathrm{Cd}}$ reconstructed. Region (3) is the disordered phase. On the left side of the dashed line, the $\mathrm{Cd}$ atoms show preferentially a local $(2 \times 1)$ ordering $\left(C_{C d}^{x}>C_{C d}^{d}\right)$, while on its right side a $c(2 \times 2)$ ordering dominates. Due to the coverage discontinuity between (1) and (2),(3), there is a coexistence regime where regions with high and low $\rho_{C d}$ coexist (4). The symbols show lines of constant $\mu$ (Same data as in Figure 2a,c) Panels (c)-(f) show typical surface snapshots. (c)-(e) correspond to grand-canonical simulations. The snapshots show surface configurations after $8 \cdot 10^{4} \cdot L N$ events. (c): $T=0.93, \mu=0.8$ (Phase 1 ), (d): $T=0.71, \mu=1$ (Phase 2), (e) $T=1.14, \mu=1$ (Phase 3 ). (f) displays a surface configuration after $2 \cdot 10^{4} \cdot L N$ events in a canonical simulation at $T=0.73, \rho_{C d}=0.35$ (Region 4, Coexistence of Phases 1 and 2). All snapshots show sections of $32 \times 32$ lattice constants of systems of size $L=N=64$. 
Figure 3 $3 \mathrm{~b}$ shows the phase diagram in the $\rho_{C d}-T$ plane. At the transition between the phases (2) and (3), $\rho_{C d}$ varies continuously. The phase transition from phase (2) to phase (1) occurs at a temperature independent chemical potential $\mu_{0}$. Therefore, there is no coverage discontinuity if temperature is varied at a constant chemical potential $\mu>\mu_{0}$ where the groundstate is a $c(2 \times 2)_{\mathrm{Cd}}$ reconstruction.

On the contrary, there is a coverage discontinuity at the phase transition between the ordered phases (1) and (2) and at the transition between phases (1) and (3). These discontinuities yield a coexistence regime (4). Here, for $T<T_{t}, c(2 \times 2)_{\mathrm{Cd}}$ and $(2 \times 1)_{\mathrm{Te}}$ reconstructed phases coexist, while for $T>T_{t}$ the $(2 \times 1)_{\mathrm{Te}}$ reconstructed phase coexists with the disordered phase. Figure $3 f$ shows a surface snapshot from a simulation which was performed at a constant Cd density $\rho_{C d}=0.35$ and a temperature $T=0.73$. This is a typical surface configuration in the regime where the ordered phases (1) and (2) coexist. The system is separated in two phases, one with a high $\rho_{C d}$ and a $c(2 \times 2)_{\mathrm{Cd}}$ reconstruction, and another one with a $(2 \times 1)_{\text {Te }}$ reconstruction and a low concentration of Cd adatoms. The local values of $\rho_{C d}$ in both regions are given by the left and the right boundary of the coexistence regime. At low temperature one obtains $\rho_{C d} \sim 0.5$ in the $c(2 \times 2)_{\mathrm{Cd}}$ phase and $\rho_{C d} \sim 0$ in the $(2 \times 1)_{\mathrm{Te}}$ phase. At temperatures $T \gtrsim 0.6, \rho_{C d}$ in the $(2 \times 1)_{\mathrm{Te}}$ phase increases strongly with $T$ and obtains its maximal value $\rho_{C d}^{1, \max }=0.23$ at $T=0.98$. At even higher temperature it decreases with $T$ and becomes zero at $T_{c}^{1}$. On the contrary, $\rho_{C d}$ in the $c(2 \times 2)_{\mathrm{Cd}}$ phase remains high for $T<T_{t}$. At $T>T_{t}$, the Cd-rich phase is disordered. Then, $\rho_{C d}$ at the right boundary of the coexistence regime decreases with $T$. At $T_{c}^{1}$, it becomes zero and the coexistence regime disappears. The dashed line in figure $3 \mathrm{a}$ marks the values of $\rho_{C d}$ at which $C_{C d}^{x}=C_{C d}^{d}$. For smaller coverages, $C_{C d}^{x}>C_{C d}^{d}$ such that the local ordering of the $\mathrm{Cd}$ atoms is dominated by a $(2 \times 1)$ arrangement, while for greater coverages, $C_{C d}^{x}<C_{C d}^{d}$. The values of $\rho_{C d}$ which have been measured in the simulations shown in figure 2 are plotted in the phase diagram as examples of lines of constant chemical potential. The loci of the phase transitions and the point where $C_{C d}^{x}=C_{C d}^{d}($ at $\mu=1)$ are in good agreement with the results of the transfer matrix extrapolation.

\subsubsection{Canonical ensemble}

The temperature dependent behaviour of the model at a constant $\mathrm{Cd}$ adatom density $\rho_{C d}=0.35$ is shown in figure 4 . This is an example which shows the typical behaviour of the model under the conditions of phase separation. At low temperature, most of the $\mathrm{Cd}$ atoms are concentrated in a $\mathrm{Cd}$ rich phase with a $c(2 \times 2)_{\mathrm{Cd}}$ reconstruction. The remaining area of the system is covered with a $(2 \times 1)_{\mathrm{Te}}$ reconstructed phase. Since both phases are long-range ordered, we obtain large values of the order parameters $M_{C d}^{(2 \times 2)}$ and $M_{D}^{(2 \times 1)}$ and the corresponding correlations. The fraction of Cd atoms which occupy sites in the Te rich phase yields nonzero values of the order parameter $M_{C d}^{(2 \times 1)}$. As temperature increases, more and more $\mathrm{Cd}$ atoms pass into the Te rich phase. This yields an increase in $C_{C d}^{x}$ and $M_{C d}^{(2 \times 1)}$ and a decrease of $C_{C d}^{d}$ and $M_{C d}^{(2 \times 2)}$ due to the $(2 \times 1)$ ordering of the Cd atoms in the Te rich phase. At the temperature $T_{t}$, the $\mathrm{Cd}$ rich phase undergoes the order-disorder transition where $M_{C d}^{(2 \times 2)}$ drops to zero. At this phase transition, the fraction of Cd atoms which are incorporated in locally $(2 \times 1)$ ordered configurations in the Cd rich phase increases. Thus, there are two independent effects which lead to a dominance of $C_{C d}^{x}$ over $C_{C d}^{d}$ at higher temperature: The specific shape of the left boundary of the coexistence regime and the order-disorder transition of the $\mathrm{Cd}$ rich phase. At a temperature $T_{c}^{4}$, the system is leaving the coexistence region into phase (3). At this phase transition, the separation between a 

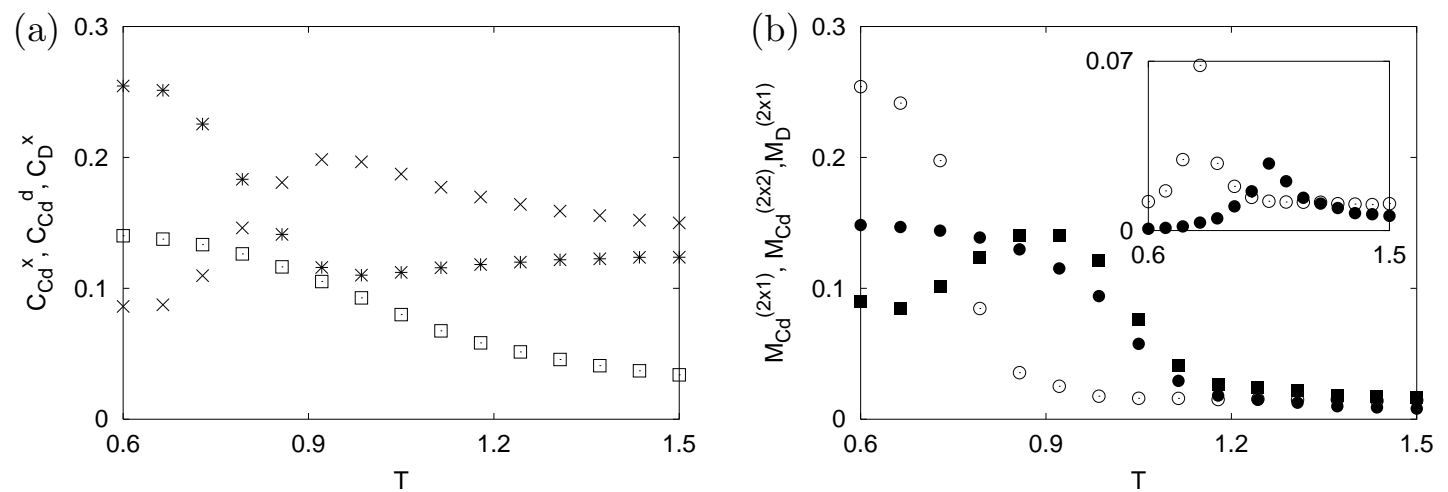

Figure 4: Results of a simulation of a $64 \times 64$ system at conserved $\rho_{C d}=0.35$ and couplings $\epsilon_{d}=\epsilon_{b}=-1, \epsilon_{x}=\epsilon_{t}=-1.9 .10^{4} \cdot L N$ events have been performed both for equilibration and for data sampling. Panel (a) shows the correlations $C_{C d}^{x}(\times), C_{C d}^{d}(*)$ and $C_{d}^{x}(\bullet)$. Panel (b) shows the mean absolute of the order parameters $M_{C d}^{(2 \times 2)}(\odot), M_{C d}^{(2 \times 1)}(\mathbf{\square})$ and $M_{D}^{(2 \times 1)}(\bullet)$. The inset shows standard deviations of order parameters. The meaning of the symbols is the same as in the large picture.

Te rich and a $\mathrm{Cd}$ rich phase disappears, such that at high temperature the system is in a homogeneous, disordered state. The order parameters $M_{D}^{(2 \times 1)}$ and $M_{C d}^{(2 \times 1)}$ become zero and the local correlation between dimers, $C_{D}^{(2 \times 1)}$, decreases. In the simulations we have determined the critical temperatures of the phase transitions from the standard deviations of the order parameters $M_{C d}^{(2 \times 2)}$ and $M_{D}^{(2 \times 1)} . M_{C d}^{(2 \times 2)}$ is peaked at $T_{t}$, where the long range order of the Cd atoms is lost, while $M_{D}^{(2 \times 1)}$ is peaked at $T_{c}^{4}$, where the system leaves the coexistence regime and the dimers lose their long range order. We obtain $T_{t}=0.79 \pm 0.2$ and $T_{c}^{4}=1.0 \pm 0.2$, where the uncertainty is due to the temperature spacing between the single simulations. These values are systematically lower than the theoretical results of the transfer matrix extrapolation $\left(T_{t}=0.84, T_{c}^{4}=1.11\right)$. However, the theoretical results are valid in the limit of an infinite system size, where the free energy of the phase boundary can be neglected compared to that of the bulk of the phases. We have verified that the systematic deviation between theory and simulations decreases with the system size.

\subsubsection{The influence of the parameter set on the phase diagram}

Finally, we discuss the influence of the parameter set on the phase diagram. The main effect of a variation of the binding energy of the Te dimers $\epsilon_{b}$ is to shift the chemical potential at which the phase transitions occur. The influence on the shape of the phase diagram is small as long as $\epsilon_{b}$ is sufficiently large such that the density of Te atoms which are neither dimerized nor bound to $\mathrm{Cd}$ atoms is small. Due to the electron counting rule, a state where a large fraction of the Te atoms remains unbound should be irrelevant for CdTe.

A smaller energy difference $\Delta E$ between a perfect $c(2 \times 2)_{\mathrm{Cd}}$ and a perfect $(2 \times 1)_{\mathrm{Cd}}$ reconstruction increases the tendency of Cd atoms to arrange in a $2 \times 1$ order. If $\epsilon_{x}=-1.95$ and all other parameters are identical to our standard parameter set, the dashed line where $C_{C d}^{x}=C_{C d}^{d}$ is shifted to a higher $\rho_{C d} \approx 0.45$. Additionally, we obtain a higher value of the maximal $C d$ coverage in the Te rich phase $\rho_{C d}^{1, \max }=0.28$, which is achieved at a lower temperature $T=0.9$. The temperature $T_{t}=0.7$ above which the disordered 

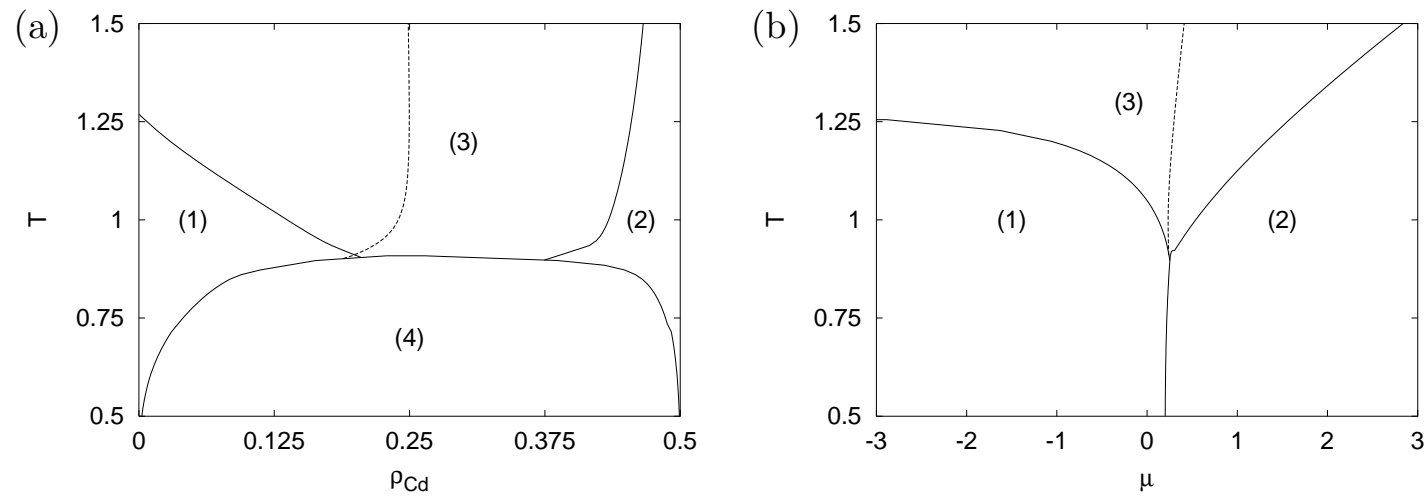

Figure 5: Phase diagram with the parameter set $\epsilon_{d}=\epsilon_{b}=-1, \epsilon_{x}=-1.6, \epsilon_{t}=-1.2$. Compared to the phase diagram shown in figure 3, the energy difference between the $c(2 \times 2)_{\mathrm{Cd}}$ and $(2 \times 1)_{\mathrm{Cd}}$ reconstruction is greater and the interaction between dimers is weaker. With these parameters, the transition between phases (1) and (3) is continuous. Panel (a) shows the phase diagram in the $\rho_{C d}-T$ plane, in panel (b) it is shown in the $\mu-T$ plane.

phase is stable is also slightly lower. In general, a greater value of $\left|\epsilon_{x}\right|$ increases both the tendency of the $\mathrm{Cd}$ atoms to arrange in a $(2 \times 1)$ order in the disordered phase and the concentration of $\mathrm{Cd}$ atoms in the $(2 \times 1)_{\mathrm{Te}}$ reconstructed phase. The temperature of the order-disorder transition in the $\mathrm{Cd}$ rich phase is lowered. Conversely, a greater energy difference between perfect $c(2 \times 2)_{\mathrm{Cd}}$ and $(2 \times 1)_{\mathrm{Cd}}$ reconstructed phases alters the temperature of this transition and leads to a preferential $c(2 \times 2)$ arrangement of the $\mathrm{Cd}$ atoms in the disordered phase.

The main effect of a variation of the interaction $\epsilon_{t}$ between Te dimers is to change the properties of the transition between the phases (1) and (3). As long as $\epsilon_{t}$ is sufficently high, the phase diagram is qualitatively similar to that shown in figure 3 . In this case, the main effect of a variation of $\epsilon_{t}$ is to shift the temperature $T_{c}^{1} \approx \epsilon_{t}$ where the $(2 \times 1)_{\text {Te }}$ phase vanishes. However, at low $\epsilon_{t}$ this transition becomes a continuous phase transition without any coverage discontinuity. As an example, in figure 3 a phase diagram with $\epsilon_{t}=-1.2$, $\epsilon_{b}=\epsilon_{d}=-1$ and $\epsilon_{x}=-1.6$ is shown. The coexistence regime (4) vanishes at $T_{t}=0.9$ such that there is no phase separation between the long-range ordered $(2 \times 1)_{\text {Te }}$ phase and the disordered phase. This parameter set yields a comparatively high $\Delta E$. Therefore, the line where $C_{C d}^{x}=C_{C d}^{d}$ is at relatively low Cd coverages. In contrast to the situation at large $\epsilon_{t}$, the chemical potential at which the transition from phase (1) to phase (2) occurs is not independent of temperature. Instead, the transition is at slightly greater $\mu$ at higher temperature. In consequence, there is a small range $0.2<\mu<0.27$ where there is a phase transition from phase (2) to phase (1) if $T$ is increased at constant chemical potential.

\section{Comparison with experimental results}

The results presented in section 3.4 suggest an interpretation of the experimentally observed crossover from a $c(2 \times 2)_{\mathrm{Cd}}$ reconstruction to a $(2 \times 1)_{\mathrm{Cd}}$ reconstruction as an accompanying effect of an order-disorder phase transition. At low temperature, there is a long-range ordered $\mathrm{Cd}$-rich phase with a $c(2 \times 2)_{\mathrm{Cd}}$ reconstruction. At a critical temperature, the $\mathrm{Cd}$ atoms lose their long-range order and arrange preferentially in a $(2 \times 1)$ pattern. This picture is consistent with the experimental observation of small domains in 
the $(2 \times 1)_{\text {Cd }}$ reconstruction [3] which indicate a high degree of disorder.

Strictly speaking, a CdTe surface under vacuum is not in thermal equilibrium. At the temperature of the $c(2 \times 2)_{\mathrm{Cd}}-(2 \times 1)_{\mathrm{Cd}}$ transition, sublimation plays an important role. However, in a previous publication [23] we have shown that the basic features of a simplified version of our model which neglects Te dimerization [12] are preserved under the conditions of step flow sublimation. This is the dominant sublimation mechanism for CdTe (001) 24]. Sublimation is slow enough to permit a local equilibration of the terminating layer which justifies the application of equilibrium thermodynamics. However, the $\mathrm{Cd}$ coverage $\rho_{C d}$ is determined by the sublimation process. Therefore, within the limit of an equilibrium model it is not possible to calculate the path in the phase diagram that CdTe follows.

Auger measurements [3] yield $\rho_{C d} \approx 0.35$ at the transition temperature in vacuum. This suggests that the system is in the coexistence regime for a wide range of temperatures. Then, the behaviour of CdTe in vacuum should be similar to our results at constant $\rho_{C d}=0.35$ (section 3.4.3). Electron diffraction techniques investigate large regions on the surface and hence yield averages over all coexisting phases. Consequently, it should be reasonable to compare results of these experiments with quantities which are averaged over the whole system in our canonical simulations. As discussed in section 3.4.3, there are two independent effects which lead to a clear dominance of the arrangement of $\mathrm{Cd}$ atoms in rows: the order-disorder transition in the Cd-rich phase and the fact that an increasing fraction of the $\mathrm{Cd}$ atoms is dissolved in the Te-rich phase. Electrons are diffracted both from the $\mathrm{Cd}$ atoms and the Te dimers which have $(2 \times 1)$ order. The superposition of these effects should yield the pronounced $(2 \times 1)$ diffraction peaks which have been observed in [2, 3].

In the $c(2 \times 2)_{\mathrm{Cd}}$ reconstructed phase, at temperatures well below the phase transition one frequently finds collective thermal excitations of adatoms, where a row of several $\mathrm{Cd}$ atoms is shifted by one lattice constant in the y-direction. Due to the repulsion between $\mathrm{Cd}$ atoms in this direction, this is possible only if one $\mathrm{Cd}$ atom per excitation is missing. An example is shown in figure 3 d. This effect has been observed experimentally by Seehover et. al. 25] at room temperature using STM microscopy. There is a striking similarity between figure 3 in 25] and figure $3 \mathrm{~d}$.

The phase diagram of our model explains the properties of the reconstructions of the CdTe (001) surface under an external particle flux. If the Cd coverage of the surface is increased by deposition of $\mathrm{Cd}$, the state of the system moves into regions of the phase diagram where the $\mathrm{Cd}$ atoms arrange preferentially in a $c(2 \times 2)$ pattern. Depending on temperature, this is either the ordered $c(2 \times 2)$ Cd phase $(2)$ or the region of the disorded phase (3) on the right side of the dashed line where $C_{C d}^{d}>C_{C d}^{x}$. Indeed, experiments [2, 7] have shown that an external Cd flux restores the $c(2 \times 2)_{\mathrm{Cd}}$ reconstruction at high temperatures where a $(2 \times 1)$ order is found under vacuum conditions. Clearly, a strong Te flux induces the formation of a long-range ordered $(2 \times 1)$ Te phase at temperatures below $T_{c}^{1}$.

On ZnSe, a Zn terminated $(2 \times 1)$ reconstruction has not been observed yet. Our model offers an explanation of this fact which is consistent with the results of density functional theory [9, 10, 8]. These calculations have shown, that the difference in the surface energies per $(1 \times 1)$ surface unit cell between perfect cation terminated $c(2 \times 2)$ and $(2 \times 1)$ reconstructions in ZnSe is significantly greater than in CdTe $(0.03 \mathrm{eV}$ versus $0.008 \mathrm{eV})$. In our model, this greater energy difference corresponds to a smaller value of $\left|\epsilon_{x}\right|$ which shifts the line where $C_{C d}^{x}=C_{C d}^{d}$ to smaller coverages. Consequently, a $(2 \times 2)_{\mathrm{Zn}}$ arrangement dominates in a much wider range of coverages. 
Wolframm et. al. [4] have measured the locus of the transition beween the $(2 \times 2)_{\mathrm{Zn}}$ reconstruction and the $(2 \times 1)_{\text {Se }}$ reconstruction as a function of temperature and the composition of an external particle flux by means of reflection high energy electron diffraction (RHEED). They find that at higher temperature a greater Se flux is required to obtain a $(2 \times 1)$ diffraction pattern. At temperatures above $450{ }^{\circ} \mathrm{C}$ no $(2 \times 1)_{\text {Se }}$ reconstruction could be observed even under extremely Se-rich conditions. This is reminiscent of our observation that the anion-rich phase (1) vanishes at a temperature $T_{c}^{1}$.

Unfortunately, the available experimental data are insufficient for a systematic fit of the model parameters. However, some rough estimates show that at least the orders of magnitude are reasonable. As discussed above, the $c(2 \times 2)_{\mathrm{Cd}}-(2 \times 1)_{\mathrm{Cd}}$ transition of CdTe in vacuum should be at a temperature close to $T_{t}$. Identifying this with the experimental value of $570 \mathrm{~K}$, we obtain the value of our energy unit $\left|\epsilon_{d}\right| \approx 0.06 \mathrm{eV}$ in physical units. This yields a value $\Delta E \approx 0.003 \mathrm{eV}$ for the difference in the surface energies of $c(2 \times 2)_{\mathrm{Cd}}$ and $(2 \times 1)_{\mathrm{Cd}}$, which is about $1 / 2$ of the value of $0.008 \mathrm{eV}$ which has been obtained by means of DFT calculations. This shows at least that the qualitative agreement between experiments and our model has been obtained in a physically reasonable region of the parameter space.

In our model, phase (1) vanishes at a temperature $T_{c}^{1} \approx\left|\epsilon_{t}\right|$. Identifying this with the value of $450^{\circ} \mathrm{C}$ which has been measured in experiments on $\mathrm{ZnSe}$, we obtain that $\epsilon_{t} \approx 0.06 \mathrm{eV}$. This is the same order of magnitude as our estimate of $\left|\epsilon_{d}\right|$ in CdTe.

These considerations suggest that it should be possible to obtain quantitative agreement between experiments and our model both for CdTe and ZnSe with values of the model parameters on the order of magnitude of a few ten meV.

\section{Acknowledgements}

We thank Wolfgang Kinzel and Richard Metzler for stimulating discussions and a critical reading of the manuscript. M.A. was supported by the Deutsche Forschungsgemeinschaft.

\section{Appendix: Algorithms for continuous time Monte Carlo sim- ulations}

We have simulated our model using continuous time Monte Carlo techniques which greatly improve the computational speed compared to simpler, Metropolis like algorithms [22]. A Markov chain of states $s$ of the system is constructed with transition rates $r_{s \rightarrow s^{\prime}}$ between states $s$ and $s^{\prime}$. This dynamics converges to a Gibbs distribution if every possible state of the system can be reached within a finite number of transition events (ergodicity) and the rates fulfil a detailed balance condition $r_{s \rightarrow s^{\prime}} / r_{s^{\prime} \rightarrow s}=\exp \left(-\left(H\left(s^{\prime}\right)-H(s)\right) / T\right)$. In every time step, one event is performed which is selected randomly with probability $p_{s \rightarrow s^{\prime}}=$ $r_{s \rightarrow s^{\prime}} / R(s)$, where $R(s)=\sum_{s^{\prime}} r_{s \rightarrow s^{\prime}}$ is the sum of transition rates of all possible events in state $s$. The physical time interval $\Delta t(s)=1 / R(s)$ which passes between subsequent events depends on the state of the system. In the calculation of thermal averages, the observables measured in state $s$ have to be weighted with $\Delta t(s)$. This algorithm requires the knowledge of the rates of all possible events in the current state of the system. If the configuration of the system is changed only locally in an event, both the selection of events and the updates of the rates of events can be done in $\mathcal{O}(\log \mathcal{N}) \mathrm{CPU}$ steps using a binary search tree. Here, $\mathcal{N}$ is the number of possible events. 
The application to grand-canonical simulations is straightforward. There are two possible events per lattice site which change the state of the site: $x_{i, j} \leftarrow\left(x_{i, j}+1\right) \bmod 3$ and $x_{i, j} \leftarrow\left(x_{i, j}+2\right) \bmod 3$. These simulations can be done with Metropolis-type rates $r_{s \rightarrow s^{\prime}}=$ $\min \left\{1, \exp \left[-\left(H\left(s^{\prime}\right)-H(s) / T\right]\right\}\right.$ or symmetrical rates $r_{s \rightarrow s^{\prime}}=\exp \left[-\left(H\left(s^{\prime}\right)-H(s)\right) /(2 T)\right]$. Both possibilities yield identical results.

A canonical simulation requires an algorithm where the number of particles is fixed. In this case, in every event one particle jumps to a different site. We choose a nonlocal dynamics where the range of particle jumps is unlimited. This yields considerably faster equilibration compared to a Kawasaki dynamics with nearest neighbour diffusion only. For simplicity, we permit only jumps to a site where the binding energy of the particle is independent of the state of its initial site, i.e. we forbid jumps to nearest and next nearest neighbour sites. If the particle jumps from site $i$ to site $j$, the energy difference between the final and the initial state is $\Delta H=\Delta H_{j}-\Delta H_{i}$, where $\Delta H_{x}$ is the energy difference of the system with site $x$ occupied and empty. The rates

$$
r_{i \rightarrow j}=\exp \left[\left(\Delta H_{i}-\Delta H_{j}\right) /(2 T)\right]
$$

fulfil the detailed balance condition. Then, the probability for a jump from site $i$ to site $j$ factorizes, i. e.

$$
p_{i \rightarrow j}=p_{i}^{-} \cdot p_{j}^{+} \quad \text { where } \quad p_{x}^{ \pm}=\frac{r_{x}^{ \pm}}{\sum_{x} r_{x}^{ \pm}} .
$$

Here, we have introduced the rate for deposition of a particle at site $x\left(r_{x}^{+}\right)$and for removal of a particle at site $x\left(r_{x}^{-}\right) \cdot r_{x}^{+}=\exp \left[-\left(\Delta H_{x}\right) /(2 T)\right]$ if site $x$ is empty and zero otherwise. Conversely, $r_{x}^{-}=\exp \left[\Delta H_{x} /(2 T)\right]$ on occupied sites and zero on empty sites. Due to this factorization property we can proceed in two steps: In the first step, we select the site $i$ from which the particle starts with probability $p_{i}^{-}$. Then, we select the site $j$ where the particle is landing with probability $p_{j}^{+}$. If the distance between site $i$ and site $j$ is $\geq 2$ the particle is moved. Otherwise, the event is rejected and the system remains unchanged. Since the number of rejected events is small on large systems, the loss of speed can be neglected.

However, in our model only the number of $\mathrm{Cd}$ atoms is fixed, while the number of $\mathrm{Te}$ dimers may change. This requires a more elaborate algorithm which uses both canonical and grand-canonical techniques. A Cd atom may jump to any site which is not occupied by a $\mathrm{Cd}$ atom. If the arrival site is occupied by a dimer, the dimer is destroyed. The removal of the $\mathrm{Cd}$ atom at the starting site creates a pair of Te atoms. We consider both the case where these Te atoms dimerize immediately and the case where they remain unbound. Additionally, Te dimers may break up and dimers may be created on empty sites. In the following, these processes will be denoted as "dimer flips". In each timestep, a random number is drawn to decide whether a Cd jump with immediate formation of a dimer at the starting site, a Cd jump without dimerization or a dimer flip will occur. The probabilities are proportional to the sums of the rates of the processes in each group. A $L \times N$ system contains $\mathcal{O}(L N) \mathrm{Cd}$ atoms and Te dimers. Thus, there are $\mathcal{O}(L N)$ dimer flip events. Since there are $\mathcal{O}(L N)$ possible arrival sites for each $\mathrm{Cd}$ atom, there are $\mathcal{O}\left(L^{2} N^{2}\right)$ Cd jump events. To keep the ratio of dimer flips and $\mathrm{Cd}$ jumps independent of the system size, the dimer flips have been weighted with a prefactor $L N$. Then, one event in the selected group is perfomed. For dimer flips, this is done with the grand-canonical algorithm while $\mathrm{Cd}$ jumps are performed by the canonical two-step algorithm.

\section{References}


[1] I. Suemune, A. Ishibashi (Eds.), Proceedings of the Ninth International Conference on II-VI Compounds, Kyoto 1999, J. Cryst. Growth 214-215 (2000)

[2] S. Tatarenko, B. Daudin, D. Brun, V. Etgens, M. B. Veron. Phys. Rev. B 50, 18479 (1994)

[3] H. Neureiter, S. Tatarenko, S. Spranger, M. Sokolowski. Phys. Rev. B 62, 2542 (2000)

[4] D. Wolfframm, D. A. Evans, D.I. Westwood, J. Riley. Journ. Cryst. Growth 216, 119 (2000)

[5] B. Daudin, D. Brun - Le Cunff, S. Tatarenko. Suf. Sci. 352-354, 99 (1994)

[6] M. B. Veron, A. Arnoult, B. Daudin, S. Tatarenko. Phys. Rev. B 54 (8), R5267 (1996)

[7] J. Cibert, S. Tatarenko. Defect and Diffusion Forum 150-151, 1 (1997) and references therein.

[8] S. Gundel, A. Fleszar, W. Faschinger, W. Hanke. Phys. Rev. B 59, 15261 (1999)

[9] A. Garcia, J. Northrup. J. Vac. Sci. Technol. B 12, 2678 (1994)

[10] C. H. Park, D. J. Chadi. Phys. Rev. B 49, 16647 (1994)

[11] V. P. LaBella, D. W. Bullock, M. Anser, Z. Ding, C. Emery, L. Bellaiche, P. M. Thibado. Phys. Rev. Lett. 84 (18), 4152 (2000)

[12] M. Biehl, M. Ahr, W. Kinzel, M. Sokolowski, T. Volkmann. Europhys. Lett. 53 (2), $169(2001)$

[13] M. Schick. in Phase Transitions in Surface Films. by J. G. Dash and J. Ruvalds (eds.) Plenum Press, New York (1980)

[14] W. Kinzel, W. Selke, K. Binder. Surf. Sci. 121, 13 (1982) and W. Selke, K. Binder, W. Kinzel. Surf. Sci. 125, 74 (1983)

[15] M. D. Pashley. Phys. Rev. B 40, 10481 (1989)

[16] W. A. Harrison. J. Vac. Sci. Technol. B 16, 1492 (1979)

[17] H. J. Cornelissen, D. A. Gammack, R. J. Dalby. J. Vac. Sci. Technol. B 6 (2), 769 (1988)

[18] W. J. Camp, M. E. Fisher. Phys. Rev. B 6 (3), 946 (1972)

[19] P. Nightingale. J. Appl. Phys. 53 (11), 7927 (1982);

[20] N. C. Bartelt, T.L. Einstein, L.D. Roelofs. Phys. Rev. B 34, 1616 (1986)

[21] P. Nightingale Phenomenological Renormalization Group Theory. PhD Thesis, Univ. of Amsterdam 1978

[22] M. E. Newman, G. T. Barkema. Monte Carlo Methods in Statistical Physics. Clarendon Press, Oxford (1999)

[23] M. Ahr, M. Biehl. Surf. Sci. 488 L553 (2001) 
[24] H. Neureiter, S. Schinzer, W. Kinzel, S. Tatarenko, M. Sokolowski. Phys. Rev. B 61 (8), 5408 (2000)

[25] L. Seehover, G. Falkenberg, R. L. Johnson, V. H. Etgens, S. Tatarenko, D. Brun, B. Daudin. Appl. Phys. Lett. 67 (12), 1680 (1995) 